See discussions, stats, and author profiles for this publication at: https://www.researchgate.net/publication/321015449

\title{
Right Brodmann area 18 predicts tremor arrest after Vim radiosurgery: a voxel-based morphometry study
}

Article in Acta Neurochirurgica - November 2017

DOI: 10.1007/500701-017-3391-x

\section{CITATIONS}

15

12 authors, including:

Constantin Tuleasca

Centre Hospitalier Régional Universitaire de Lille

365 PUBLICATIONS 565 CITATIONS

SEE PROFILE

Antoine Verge

University of Lorraine

127 PUBLICATIONS 650 CITATIONS

SEE PROFILE
READS

68

Dimitri Van De Ville

École Polytechnique Fédérale de Lausanne

660 PUBLICATIONS 9,665 CITATIONS

SEE PROFILE

Nadine Girard

Assistance Publique Hôpitaux de Marseille

421 PUBLICATIONS 4,773 CITATIONS

SEE PROFILE

Some of the authors of this publication are also working on these related projects:

Combined approaches View project

Project Radiosurgical treatment of meningiomas View project 


\section{Right Brodmann area 18 predicts tremor arrest after Vim radiosurgery: a voxel-based morphometry study}

Constantin Tuleasca ${ }^{1,3,9}$, MD, Tatiana Witjas ${ }^{4,5}$, MD, Dimitri van de Ville*11, PHD, Elena Najdenovska ${ }^{2}$, MSc, Antoine Verger ${ }^{6}$, MD, Nadine Girard ${ }^{7}$, MD-PHD, Jerome Champoudry ${ }^{8}$, MSc, Jean-Philippe Thiran ${ }^{3,9,11}$, PHD, , Meritxell Bach Cuadra ${ }^{2}$, PHD, Marc Levivier ${ }^{1,9}$, MD, PHD, Eric Guedj ${ }^{6}$, MD, AND Jean Régis ${ }^{8}$, MD

${ }^{1}$ Centre Hospitalier Universitaire Vaudois (CHUV), Neurosurgery Service and Gamma Knife Center, Lausanne, Switzerland; ${ }^{2}$ Radiology Department, Center for Biomedical Imaging (CIBM), Lausanne University Hospital (CHUV) and University of Lausanne (UNIL), Centre Hospitalier Universitaire Vaudois, Lausanne, Switzerland; ${ }^{3}$ Signal Processing Laboratory (LTS 5), Ecole Polytechnique Fédérale de Lausanne (EPFL), Switzerland; ${ }^{4}$ Department of Neurology and Movement Disorders, Assistance Publique-Hôpitaux de Marseille, Aix-Marseille Université, Timone University Hospital, France; ${ }^{5}$ Institut de Neurosciences de la Timone, UMR 7289, CNRS Aix-Marseille Université, Marseille, France; ${ }^{6}$ Department of Nuclear Medicine, Assistance Publique-Hôpitaux de Marseille, Aix-Marseille Université, Timone University Hospital, France;Institut de Neurosciences de la

Timone, UMR 7289, CNRS Aix-Marseille Université, Marseille, France;CERIMED, Aix-Marseille Université, Marseille, France; ${ }^{7}$ AMU, CRMBM UMR CNRS 7339, Faculté de Médecine et APHM, Hopital Timone,

Department of Diagnostic and Interventionnal Neuroradiology, Marseille; ${ }^{8}$ Stereotactic and Functional Neurosurgery Service and Gamma Knife Unit, CHU Timone, Marseille, France; ${ }^{9}$ University of Lausanne, Faculty of Biology and Medicine; ${ }^{10}$ Medical Image Processing Laboratory, Ecole Polytechnique Fédérale de Lausanne (EPFL), Switzerland ; ${ }^{11}$ University of Geneva, Faculty of Medicine; ${ }^{12}$ Centre Hospitalier Universitaire Vaudois, Radiology Department

* the authors equally contributed to the present study

\section{Corresponding author:}

Constantin Tuleasca, MD, MD-PHD candidate, Centre Hospitalier Universitaire Vaudois, Neurosurgery Service and Gamma Knife Center, Rue du Bugnon 44-46, BH-08, CH-1011, Lausanne, Switzerland; Tel: +41-21-314-26-02; Fax: +41-21-314-11-99; e-mail: constantin.tuleasca@gmail.com 


\section{Abstract}

Introduction: Drug-resistant essential tremor (ET) can benefit from open standard stereotactic procedures, such as deep-brain stimulation or radiofrequency thalamotomy. Nonsurgical candidates can be offered either high-focused ultrasound (HIFU) or radiosurgery (RS). All aim to target the same thalamic site, ventro-intermediate nucleus (e.g. Vim). The mechanisms by which tremor stops after Vim RS or HIFU remain unknown. We used voxelbased morphometry (VBM) on pretherapeutic neuroimaging data and assessed which anatomical site would best correlate with tremor arrest 1 year after the procedure.

Methods: Fifty-two patients (30 male, 22 female; mean age 71.6 years, range 49-82) with right-sided ET benefited from left unilateral Vim RS in Marseille, France. Targeting was performed in a uniform manner, using $130 \mathrm{~Gy}$ and single 4-mm collimator. Neurological (pretherapeutic and 1 year after) and neuroimaging (baseline) assessment were completed. Tremor score on treated hand (TSTH) at 1 year after Vim RS was included in a Statistical Parametric Mapping Anova model as a continuous variable, with pretherapeutic neuroimaging data. Pretherapeutic grey matter density (GMD) was correlated with TSTH improvement. No a priori hypothesis was used in the statistical model.

Results: The only statistically significant region was right Brodmann area (BA) 18 (visual association area V2, $\mathrm{p}=0.05$, cluster size $\mathrm{K}_{\mathrm{c}}=71$ ). Higher baseline GMD correlated with better TSTH improvement (Spearmann's rank correlation coefficient $=0.002)$.

Conclusions: Routine baseline structural neuroimaging predicts TSTH improvement 1 year after Vim RS. The relevant anatomical area is right visual association cortex (BA 18, V2). The question whether visual areas should be included in the targeting remains opened.

Key words: tremor, ventro-intermediate nucleus, voxel-based morphometry, visual association area, radiosurgery, thalamotomy 


\section{INTRODUCTION}

Tremor characterizes one of the clearest consequences of unusual synchronization within the motor network. Although many studies attempted understanding physiopathology of tremor[17, 18, 44], its underlying mechanisms remain largely undiscovered. Essential tremor (ET) is the most common movement disorder[27]. It has been beforehand promoted that in the context of ET, thalamic neurons display discharging patterns correlated with tremor, predominantly in the cerebellar input-receiving area (mainly dentate nucleus) and further in contralateral red nucleus, thalamic ventro-intermediate nucleus (Vim) and precentral gyrus[21]. The "tremor-network" encompasses so the cerebello-thalamo-cortical loop[18]. It has been also promoted that the inferior olivary nucleus might produce an abnormal rhythmic output, which further affects synchronization of Purkinje cell firing[19], passively propagated through the cerebello-thalamic tract $[5,30]$, and lastly regulating motor activity[31].

Drug resistant ET can benefit from standard stereotactic procedures, including deepbrain stimulation (DBS)[3] by the means of an electrode positioned in the ventral thalamus (the ventro-intermediate nucleus, i.e. Vim) or radiofrequency thalamotomy[28]. Both have the advantage of the intraoperative confirmation of targeting, with the possibility of adjusting the placement of the electrode if necessary, which can be performed on the basis of intraoperative electrophysiology and clinical response; furthermore, they provide immediate postoperative clinical effect, with regard to alleviation of the tremor[6, 20]. Non-surgical candidates can be proposed radiosurgery (RS; e.g. Vim RS, also named Gamma Knife thalamotomy) $[8,23,41]$ or high-focused ultrasound (HIFU) $[14,15,26]$. They aim at the same target (e.g. Vim). The mechanisms by which RS and HIFU produce tremor arrest remain unknown. Radiosurgical targeting is indirect and clinical effect installs gradually, up to one year after the procedure[41], unlike open surgery. It has been previously hypothesized that, beside a purely lesionning effect, Vim RS might have a neuromodulatory one[33].

The advances in neuroimaging procedures have provided new understanding onto the pathophysiology of tremor[34]. Computational approaches for analyzing high-resolution structural MRI provide a powerful and non-invasive tool for characterizing individual and/or group differences in brain anatomy[1]. With regard to structural MRI, voxel-based morphometry (VBM) is a well-established and robust methodology, allowing an insight to depict structural brain features. It uses the routinely acquired $\mathrm{T} 1$ weighted imaging $(\mathrm{T} 1 \mathrm{w})$, to study volume subparts, gry or white matter density[1]. For ET, VBM has been used both at 
1.5 and 3 Tesla (T), with large spectrum of controversial findings[2, 4, 7, 10-12, 25, 29, 32$]$. However, all these studies have compared ET cases versus a group of healthy controls.

In the present study, we use pretherapeutic VBM assessment and correlate it with tremor score on the treated hand improvement (TSTH) at 1 year after Vim RS. We used no a priori assumption. We hypothesized (based on previous work from our group, unpublished data), that visual areas might play a role in tremor alleviation and/or arrest after Vim RS.

\section{METHODS}

\section{Subjects}

We analyzed 52 patients (30 male, 22 female; mean age 71.6 years, range $49-82$, standard deviation 6.9). All had severe refractory right-dominant in right-handed ET, treated with unilateral left Vim RS, in Marseille, France. All had given informed written consent. The study was approved by local ethics committee (in compliance with the national legislation and the Declaration of Helsinki). All were part of a research protocol, aiming at the understanding of Vim RS radiobiology. Inclusion and exclusion criteria can be seen in table 1. Essential tremor has been defined according to Elble et al.[13]. The indication for Vim RS rather than DBS were medical co-morbidities drug-resistance, advanced age or patient's refusal.

\section{Ventro-intermediate nucleus radiosurgery procedure}

All Vim RS were all performed by same neurosurgeon (JR), in Marseille, France, using a uniform procedure: Leksell ${ }^{\circledR}$ coordinate G Frame (Elekta AB, Stockholm, Sweden) application, under local anesthesia[41], stereotactic CT and MRI, indirect targeting using Guiot's diagram $(2.5 \mathrm{~mm}$ above the AC-PC line, $11 \mathrm{~mm}$ lateral to the wall of the third ventricle, and at a mean of $7.3 \mathrm{~mm}$ in front of the PC (3.8-9.8; SD: 1.3)). A unique 4-mm isocenter was used, and a maximal prescription dose of $130 \mathrm{~Gy}$.

\section{Clinical evaluation and outcome measures after Vim radiosurgery}

The same neurologist (TW) performed all clinical assessment, at baseline and 1 year after Vim RS, to account for delayed clinical effect[41]. Tremor score on the treated hand (TSTH) was assessed according to the Fahn-Tolosa-Marin Tremor Rating Scale[36]. At the time of study, no patient was under medication.

\section{MRI pretherapeutic acquisition}


All imaging was obtained on a 3-T magnetic resonance imaging (MRI) scanner, SIEMENS Skyra TrioTim Scanner (Munich, Germany), with 32 channels receive array head coil. The acquisition protocol included high-resolution T1-weighted (T1w), $\mathrm{TR} / \mathrm{TE}=2300 / 2.03$, inversion time $900 \mathrm{~ms}$, isotropic voxel of $1 \mathrm{~mm}^{3}, 192$ slices. Images artefacted by movement were excluded.

\section{Data processing and analysis}

Statistical Parametric Mapping (SPM12) (Department of Neurology, London), performed with MATLAB 2014a, 2014 (MathWorks, Boston, MA, USA) was utilized for preprocessing and analyzing of the MRI data. The anatomical MR images were normalized to the Montreal Neurological Institute (MNI) atlas, with voxel-size of $2 \times 2 \times 2 \mathrm{~mm}$. The normalization procedure allows the warped images to fit into a standard template brain. Furthermore, this establishes a voxel-to-voxel correspondence, between brains of different individuals. It will enable to drive group statistics and to report the results in a standard coordinate system (e.g. MNI, which is based upon data from many individuals and is fully $3 \mathrm{D}$, with data at every voxel). They were further segmented in GM, white matter and cerebrospinal fluid. Furthermore, they were spatially smoothed using a 8-mm full-width-at-halfmaximum (FWHM) Gaussian Kernel in SPM12, to blur individual variations in gyral anatomy and to increase the signal-to-noise ratio. The smoothing procedure enables the subsequent voxel-by-voxel analysis comparable to a region of interest approach, as the voxel will contain the average concentration of gray matter from around the voxel (where the voxel is characterized by the form of the smoothing kernel). The former is so often referred as to GMD. In a structural MRI of the brain, the intensity of each volumetric pixel, or voxel, relates to the density of the gray matter in that region. The VBM technique is so comparing brain structure on a voxel-by-voxel basis. After the classical preprocessing steps, the intensity values of the voxels are compared, to identify localized differences in GMD.

SPM 12 was used to create a flexible factorial analysis of variance (Anova) model. Pretherapeutic T1w imaging was used, and TSTH improvement was added as a covariate, using the corresponding continuous values. The aim was to depict the anatomical area, which correlates best with tremor stop after Vim RS. The uncorrected p value at cluster level was set at $<=0.05$ and for the peak level $<0.001$.

The SPM analysis was done by medical staff (CT, DVDV, EG) not involved in treatment indication, Vim RS procedure or follow-up evaluation. Grey matter density (GMD) was further extracted from statistically significant regions. For correlation between GMD and 
TSTH improvement, STATA version 11 (STATA Corp LLC, College Stations, TX, USA) was used, and $\mathrm{p}$ values evaluated with Spearman correlation coefficient.

\section{RESULTS}

One year overall improvement in TSTH in the present population was $67.8 \%$ (range 0 $100 \%$, standard deviation 32.9). No side effect was encountered. The vast majority of the patients presented a left thalamic "cocarde" lesion on follow-up MRI, which is classically considered associated with the clinical response[37, 41]. However, four patients, with severe pretherapeutic tremor were considered clinical non-responders but had a visible thalamic lesion on follow-up MRI, while eight patients were considered clinical responders and had a smaller lesion on follow-up MRI.

\section{Right visual association area (Brodmann area 18, V2) predicts tremor alleviation}

The only statistically significant cluster was right Brodmann area (BA) 18 (visual association area V2; MNI 12, -74, $-4 ; \mathrm{p}_{\text {uncor }}=0.05, \mathrm{~K}_{\mathrm{c}}=71$; figure 1). Higher pretherapeutic GMD correlated with better TSTH improvement (Spearmann=0.002; figure 1).

\section{DISCUSSION}

To the best of our knowledge, we performed the first VBM analysis endeavoring to predict which structural changes and/or adaptations in patients with drug-resistant ET would best correlate with one-year outcome (e.g. tremor score on the treated hand) after Vim RS. We report that right Brodmann area 18 best correlates with TSTH improvement. Furthermore, higher baseline grey matter density within this region associates with better TSTH improvement.

Visual association area V2, also called the prestriate cortex, is the second major area in the visual cortex. This region receives connections from the V1 and sends strong connections to the V3, V4 and V5 areas. Most of the neurons are regulated to simple visual features such as orientation, spatial frequency, size, color and shape. It is mainly involved in visuo-spatial information processing[39]. Other roles include detection of light intensity, detection of patterns, discrimination of finger gestures or orientation-selective attention[24]. In fact, according to some functional studies, it participates additionally in visual priming and visual attention[9].

The previous studies related to VBM and ET patients have mainly compared the ET with healthy controls, and revealed inconsistent results (at 1.5 and $3 \mathrm{~T}[2,4,7,10,11,25,32]$, 
showing atrophy, no change or volume increase in different brain areas). In the present study, it was beyond our purpose to analyze a healthy control group, as we were interested in correlating an eventual statistically significant structural feature, present pretherapeutically, to clinical response at 1 year after Vim RS.

In line with our present findings, using a VBM methodology, Daniels et al.[11] revealed a relative expansion of grey matter bilaterally in the temporo-parietal junction and the right middle occipital cortex in patients with ET as compared with healthy controls. The authors concluded that these areas, involved in higher order visuospatial processing and control, might exhibit an adaptive reorganization, and compensate skilled movements in case of tremor. In a recent functional resting-state MRI study, Jang et al.[22] evaluated the effect on brain networks of Vim HIFU in 10 patients, using graph analysis. Interestingly, the authors observed changes in functional connectivity in right lateral inferior occipital cortex. They hypothesized that a loss of strong interactions might appear or a reduced functional interactions on the way to other brain regions. Concerning the eventual distant effects of a thalamotomy procedure, structural diffusion tensor imaging (DTI) data, after HIFU thalamotomy for ET[40] showed that fractional anisotropy changes included pre- and postcentral subcortical white matter, thalamus, red nucleus, inferior olive, bilateral parts of the superior vermis or the central tegmental tract. After correction for multiple comparisons, only distant DTI changes were correlated with the clinical improvement[40].

Previous studies have postulated that visual areas must be linked to the motor ones, as a need for sensory guidance of movement of hand and fingers[16], and further so in motor regulation. Several possibilities would explain the previous: one would be a modulation by the corpus callosum (mediating tasks in which the visual cortex on one part of the body is linked to the contralateral motor cortex), a second would be a visual input to motor areas (by caudate nucleus and claustrum) and a third that cerebellum might play the essential role of calibrating the relationship between visual and somatosensory/motor information.

In a recently published paper[38], we have evaluated longitudinal GMD changes in time, between pretherapeutic state and 1 year after Vim RS. We assessed whether these structural MR changes would relate to TSTH improvement, by conventionally separating two different groups, clinically responders $(<50 \%$ improvement) and non-responders $(>=50 \%$ improvement). Furthermore, we have also appraised, whether pretherapeutic GMD, within the statistically significant clusters, would correlate with 1 year TSTH improvement, in the same study. We found that GMD was lower at baseline in the non-responders group for left BA 19 and left temporal pole (BA 38), V4, V5 and parahippocampal place area. Moreover, for the left temporal pole, higher baseline GMD predicted better TSTH improvement. 
In the present paper, we have exclusively used pretherapeutic structural $\mathrm{T} 1 \mathrm{w}$ imaging. The VBM preprocessing steps were identical. However, the research question and the statistical analysis model pertinent to this question were different. Here, we constructed a general linear model allowing predicting if an eventual pretherapeutic GMD in a specific anatomical area would relate to TSTH improvement. Of particular importance, we used TSTH as a continuous variable, without splitting of any group, as in the previous study[38]. We found that higher pretherapeutic GMD within right BA 18 predicted better TSTH improvement.

In our opinion, these findings should rather be seen as complementary, nor exclusive. Both studies point out towards the involvement of the visual association areas in tremor arrest after Vim RS. Furthermore and together, they show that lower baseline GMD in these areas (independently if right or left) and in the left temporal pole, was associated with lower TSTH improvement. Some other additional aspects should be taken into account, due to their relevance. Firstly, from methodological point of view, as previously explained, the research questions were different. Secondly, it is well known that several visual functions (e.g. detection of light intensity, feature extraction or detection of patterns etc) simultaneously activate both BA 18 and 19, which are in close anatomical location, suggesting a common brain network. Thirdly, with regards to the visual perception, it is now well acknowledged that it is not carried out in just one area, but involves many different regions of the cerebral cortex $[42,43]$, receiving input from V1. Typically, the corresponding processing of different information streams continues beyond M1. Many outputs go to V2 (e.g. BA 18), relate to motion analysis and this information is further process in V3 (e.g. BA 19) and then V5, the medial temporal (MT) cortex (part of the study already published) and medial superior temporal (MST) cortex. All these steps happen before the information moves to other visual association areas, such as the posterior parietal cortex. Fourthly, with regard to functions related to visual association cortex, electrophysiology data suggest sensitivity to motiondelineated forms[35] and its role as a differentiation point between the 'what' and 'where' visual pathways. Additional involvement is in visuospatial information processing, horizontal saccadic eye movements, orientation-selective attention and tracking visual motion patterns[42]. These functions involve both hemispheres and do not limit to a certain unilateral and individual area (no matter if V2 or V3, V4 etc). Ultimately, a possibility that was raised during time and that was already evoked here is that the cerebellum might play the crucial role of adjusting the link between visual and somatosensory/motor information[16]. This former feature might be of particular interest in ET.

We do believe that these findings, from two different studies, in which two different methodologies were used, provide rich and rather complementary information about what structural change relates to a better clinical response after Vim RS. Our research question was 
not conducted to support existing physiopathological theories and thus our findings might provide new insight. The depicted changes in relationship to tremor response might find several possible explanations. One would be that adaptive structural MR changes appear during disease course and, depending on these changes, the "reset" of the "tremor network" might be either easier or more difficult after Vim RS. A second hypothesis would see ET as a family of diseases, rather than a uniform entity. With regard to this, some of the patients, depending on their phenotype, might develop structural MRI changes making them more "sensitive" or "resistant" to Vim RS, as a trait of ET subclinical phenotype.

The advantages of the VBM analysis are related to an unbiased and objective procedure, fully automated, not based on regions of interest and which is more exploratory. Furthermore, it depicts differences and/or changes (in our study) at a global and local scale. The limitations of our study are mainly related to the absence of a blinded neurological examination and those of the VBM method (preprocessing steps, statistical challenges etc).

The role of right visual association areas depicted on routine structural pretherapeutic neuroimaging is reported for the first time as a predictor for tremor improvement after Vim RS. How the "tremor network" (e.g. the cerebello-thalamo-cortical pacemaker) modulates these structural adaptations in patients with ET remains unknown.

\section{Conclusion}

Right visual association (BA18, V2) predicts tremor arrest after Vim RS. The mechanism by which this visual area modulates the clinical response remains for the moment unknown. The question whether visual areas should be included in the targeting remains opened.

\section{References:}

1. Ashburner J, Friston KJ (2000) Voxel-based morphometry--the methods. NeuroImage 11:805-821

2. Bagepally BS, Bhatt MD, Chandran V, Saini J, Bharath RD, Vasudev MK, Prasad C, Yadav R, Pal PK (2012) Decrease in cerebral and cerebellar gray matter in essential 
tremor: a voxel-based morphometric analysis under 3T MRI. Journal of neuroimaging : official journal of the American Society of Neuroimaging 22:275-278

3. Benabid AL, Pollak P, Gao D, Hoffmann D, Limousin P, Gay E, Payen I, Benazzouz A (1996) Chronic electrical stimulation of the ventralis intermedius nucleus of the thalamus as a treatment of movement disorders. Journal of neurosurgery 84:203-214

4. Benito-Leon J, Alvarez-Linera J, Hernandez-Tamames JA, Alonso-Navarro H, Jimenez-Jimenez FJ, Louis ED (2009) Brain structural changes in essential tremor: voxelbased morphometry at 3-Tesla. Journal of the neurological sciences 287:138-142

5. Bhalsing KS, Saini J, Pal PK (2013) Understanding the pathophysiology of essential tremor through advanced neuroimaging: a review. J Neurol Sci 335:9-13

6. Blomstedt P, Hariz GM, Hariz MI, Koskinen LO (2007) Thalamic deep brain stimulation in the treatment of essential tremor: a long-term follow-up. British journal of neurosurgery 21:504-509

7. Buijink AW, Broersma M, van der Stouwe AM, Sharifi S, Tijssen MA, Speelman JD, Maurits NM, van Rootselaar AF (2016) Cerebellar Atrophy in Cortical Myoclonic Tremor and Not in Hereditary Essential Tremor-a Voxel-Based Morphometry Study. Cerebellum 15:696-704

8. Campbell AM, Glover J, Chiang VL, Gerrard J, Yu JB (2015) Gamma knife stereotactic radiosurgical thalamotomy for intractable tremor: a systematic review of the literature. Radiotherapy and oncology : journal of the European Society for Therapeutic Radiology and Oncology 114:296-301

9. Carrasco M (2011) Visual attention: the past 25 years. Vision research 51:14841525

10. Cerasa A, Messina D, Nicoletti G, Novellino F, Lanza P, Condino F, Arabia G, Salsone M, Quattrone A (2009) Cerebellar atrophy in essential tremor using an automated segmentation method. AJNR. American journal of neuroradiology 30:12401243

11. Daniels C, Peller M, Wolff S, Alfke K, Witt K, Gaser C, Jansen O, Siebner HR, Deuschl G (2006) Voxel-based morphometry shows no decreases in cerebellar gray matter volume in essential tremor. Neurology 67:1452-1456

12. Daniels JK, Gaebler M, Lamke JP, Walter H (2015) Grey matter alterations in patients with depersonalization disorder: a voxel-based morphometry study. Journal of psychiatry \& neuroscience : JPN 40:19-27

13. Elble RJ (2000) Diagnostic criteria for essential tremor and differential diagnosis. Neurology 54:S2-6

14. Elias WJ, Huss D, Voss T, Loomba J, Khaled M, Zadicario E, Frysinger RC, Sperling SA, Wylie S, Monteith SJ, Druzgal J, Shah BB, Harrison M, Wintermark M (2013) A pilot study of focused ultrasound thalamotomy for essential tremor. The New England journal of medicine 369:640-648

15. Elias WJ, Lipsman N, Ondo WG, Ghanouni P, Kim YG, Lee W, Schwartz M, Hynynen K, Lozano AM, Shah BB, Huss D, Dallapiazza RF, Gwinn R, Witt J, Ro S, Eisenberg HM, Fishman PS, Gandhi D, Halpern CH, Chuang R, Butts Pauly K, Tierney TS, Hayes MT, Cosgrove GR, Yamaguchi T, Abe K, Taira T, Chang JW (2016) A Randomized Trial of Focused Ultrasound Thalamotomy for Essential Tremor. The New England journal of medicine 375:730-739

16. Glickstein M (2000) How are visual areas of the brain connected to motor areas for the sensory guidance of movement? Trends in neurosciences 23:613-617

17. Hallett M (2012) Parkinson's disease tremor: pathophysiology. Parkinsonism \& related disorders 18 Suppl 1:S85-86 
18. Hallett M (2014) Tremor: pathophysiology. Parkinsonism \& related disorders 20 Suppl 1:S118-122

19. Hansel C (2009) Reading the clock: how Purkinje cells decode the phase of olivary oscillations. Neuron 62:308-309

20. Hariz GM, Lindberg M, Bergenheim AT (2002) Impact of thalamic deep brain stimulation on disability and health-related quality of life in patients with essential tremor. Journal of neurology, neurosurgery, and psychiatry 72:47-52

21. Hua SE, Lenz FA (2005) Posture-related oscillations in human cerebellar thalamus in essential tremor are enabled by voluntary motor circuits. Journal of neurophysiology 93:117-127

22. Jang C, Park HJ, Chang WS, Pae C, Chang JW (2016) Immediate and Longitudinal Alterations of Functional Networks after Thalamotomy in Essential Tremor. Frontiers in neurology 7:184

23. Kondziolka D, Ong JG, Lee JY, Moore RY, Flickinger JC, Lunsford LD (2008) Gamma Knife thalamotomy for essential tremor. Journal of neurosurgery 108:111-117

24. Larsson J, Landy MS, Heeger DJ (2006) Orientation-selective adaptation to firstand second-order patterns in human visual cortex. Journal of neurophysiology 95:862881

25. Lin CH, Chen CM, Lu MK, Tsai CH, Chiou JC, Liao JR, Duann JR (2013) VBM Reveals Brain Volume Differences between Parkinson's Disease and Essential Tremor Patients. Frontiers in human neuroscience 7:247

26. Lipsman N, Schwartz ML, Huang Y, Lee L, Sankar T, Chapman M, Hynynen K, Lozano AM (2013) MR-guided focused ultrasound thalamotomy for essential tremor: a proof-of-concept study. The Lancet. Neurology 12:462-468

27. Louis ED (2005) Essential tremor. The Lancet. Neurology 4:100-110

28. Nagaseki Y, Shibazaki T, Hirai T, Kawashima Y, Hirato M, Wada H, Miyazaki M, Ohye C (1986) Long-term follow-up results of selective VIM-thalamotomy. Journal of neurosurgery 65:296-302

29. Nicoletti V, Cecchi P, Frosini D, Pesaresi I, Fabbri S, Diciotti S, Bonuccelli U, Cosottini M, Ceravolo R (2015) Morphometric and functional MRI changes in essential tremor with and without resting tremor. Journal of neurology 262:719-728

30. Passamonti L, Novellino F, Cerasa A, Chiriaco C, Rocca F, Matina MS, Fera F, Quattrone A (2011) Altered cortical-cerebellar circuits during verbal working memory in essential tremor. Brain 134:2274-2286

31. Popa T, Russo M, Vidailhet M, Roze E, Lehericy S, Bonnet C, Apartis E, Legrand AP, Marais L, Meunier S, Gallea C (2013) Cerebellar rTMS stimulation may induce prolonged clinical benefits in essential tremor, and subjacent changes in functional connectivity: an open label trial. Brain stimulation 6:175-179

32. Quattrone A, Cerasa A, Messina D, Nicoletti G, Hagberg GE, Lemieux L, Novellino F, Lanza P, Arabia G, Salsone M (2008) Essential head tremor is associated with cerebellar vermis atrophy: a volumetric and voxel-based morphometry MR imaging study. AJNR. American journal of neuroradiology 29:1692-1697

33. Regis J, Carron R, Park M (2010) Is radiosurgery a neuromodulation therapy? : A 2009 Fabrikant award lecture. Journal of neuro-oncology 98:155-162

34. Sharifi S, Nederveen AJ, Booij J, van Rootselaar AF (2014) Neuroimaging essentials in essential tremor: a systematic review. NeuroImage. Clinical 5:217-231

35. Tardif E, Richer L, Bergeron A, Lepore F, Guillemot JP (1997) Spatial resolution and contrast sensitivity of single neurons in area 19 of split-chiasm cats: a comparison with primary visual cortex. The European journal of neuroscience 9:1929-1939 
36. Tolosa E, Pena J (1988) Involuntary vocalizations in movement disorders. Advances in neurology 49:343-363

37. Tuleasca C, Pralong E, Najdenovska E, Cuadra MB, Marques JRF, Vingerhoets F, Regis J, Bloch J, Levivier M (2017) Deep brain stimulation after previous gamma knife thalamotomy of the Vim for essential tremor is feasible! Clinical, electrophysiological and radiological findings. Acta neurochirurgica 159:1371-1373

38. Tuleasca C, Witjas T, Najdenovska E, Verger A, Girard N, Champoudry J, Thiran JP, Van de Ville D, Bach Cuadra M, Levivier M, Guedj E, Régis J (2017, DOI 10.1007/s00701017-3317-7) Assessing the clinical outcome of Vim radiosurgery with voxel-based morphometry: visual areas are linked with tremor arrest! Acta neurochirurgica

39. Waberski TD, Gobbele R, Lamberty K, Buchner H, Marshall JC, Fink GR (2008) Timing of visuo-spatial information processing: electrical source imaging related to line bisection judgements. Neuropsychologia 46:1201-1210

40. Wintermark M, Huss DS, Shah BB, Tustison N, Druzgal TJ, Kassell N, Elias WJ (2014) Thalamic connectivity in patients with essential tremor treated with MR imaging-guided focused ultrasound: in vivo fiber tracking by using diffusion-tensor MR imaging. Radiology 272:202-209

41. Witjas T, Carron R, Krack P, Eusebio A, Vaugoyeau M, Hariz M, Azulay JP, Regis J (2015) A prospective single-blind study of Gamma Knife thalamotomy for tremor. Neurology 85:1562-1568

42. Zeki S (1993) The visual association cortex. Current opinion in neurobiology 3:155-159

43. Zeki S, Watson JD, Lueck CJ, Friston KJ, Kennard C, Frackowiak RS (1991) A direct demonstration of functional specialization in human visual cortex. The Journal of neuroscience : the official journal of the Society for Neuroscience 11:641-649

44. Zesiewicz TA, Elble R, Louis ED, Hauser RA, Sullivan KL, Dewey RB, Jr., Ondo WG, Gronseth GS, Weiner WJ, Quality Standards Subcommittee of the American Academy of N (2005) Practice parameter: therapies for essential tremor: report of the Quality Standards Subcommittee of the American Academy of Neurology. Neurology 64:20082020

Funding: This work has been supported by the Timone University Hospital, the Swiss National Science Foundation SNSF-205321-157040, the Centre d'Imagerie BioMédicale (CIBM) of the University of Lausanne (UNIL), the University of Geneva (UniGe), the Centre Hospitalier Universitaire Vaudois (CHUV) and the Leenaards and Jeantet Foundations. This work has been carried out thanks to the support of the A*MIDEX project ( ${ }^{\circ}$ ANR-11-IDEX0001-02) funded by the «Investissements d'Avenir » French Government program, managed by the French National Research Agency (ANR).

Conflict of Interest: All authors certify that they have no affiliations, with or involvement in any organization or entity with any financial interest (such as honoraria; educational grants; participation in speakers' bureaus; membership, employment, consultancies, stock ownership, or other equity interest; and expert testimony or patent-licensing arrangements), or nonfinancial interest (such as personal or professional relationships, affiliations, knowledge or 
beliefs) in the subject matter or materials discussed in this manuscript.

Ethical approval: The study was approved by local ethics committee of Timone University Hospital, Marseille, France. Patient consent was acquired in all cases.

Figure 1: The only statistically significant cluster was right Brodmann area (BA) 18 (visual association area V2). In the upper part can be seen an illustration of the cluster in axial, coronal and sagittal plane. Below, a scatterplot is correlating the baseline GMD with TSTH improvement (Spearmann=0.002). At the bottom part it is displayed the SPM result at cluster and peak level.

Table 1 : patient inclusion and exclusion criteria 